\title{
A pesquisa blue sky como alternativa para potencializar a criatividade
}

\author{
The Blue Sky Research as an alternative to boost creativity
}

DAL PAI, Dinara; Mestre; Universidade do Vale do Rio dos Sinos

dinadalpai@hotmail.com

\begin{abstract}
Resumo
Considerando a importância estratégica da inovação no contexto do mercado contemporâneo e a necessidade de reformular os métodos utilizados em projetos convencionais, este artigo discute a relevância da pesquisa blue sky durante o processo de desenvolvimento de novos produtos. Para tanto, foram abordados temas como o contexto de gerenciamento de projetos e a gestão da criatividade. Tem sido demonstrado pela literatura que o design estratégico pode contribuir significativamente para o processo de desenvolvimento de novos produtos, dividindo-o em duas etapas: o metaprojeto e o projeto. Assim, o metaprojeto é apresentado como um estágio importante, pois permite uma reflexão sobre os direcionamentos do projeto. Nesse contexto, a pesquisa blue sky aparece como uma ferramenta metaprojetual que busca estimular a criatividade por meio do uso de imagens, favorecendo a comunicação entre a equipe e a troca de conhecimentos que tendem a aumentar a criatividade e a inovação.
\end{abstract}

Palavras Chave: design, blue sky, criatividade, linguagem visual

\begin{abstract}
Given the strategic importance of innovation in the context of the contemporary market and the need to reformulate the methods used in conventional projects, this paper discusses the relevance of blue sky research during the development process of new products. In order to do so, issues such as the project management context and the management of creativity were addressed. It has been shown by the literature that strategic design can significantly contribute to the development process of new products by dividing it into two stages: the metaproject and the project. The metaproject is presented as an important stage as it allows a reflection on the project itself. Within this context, blue sky research appears as a metaprojectual tool that seeks to stimulate creativity by means of the use of images. Through the use of visual language, blue sky research favors the communication between the team and the exchange of knowledge that tend to enhance creativity and innovation.
\end{abstract}

Keywords: design, blue sky, creativity, visual language

\section{Introdução}

A grande proliferação dos bens industrializados e a consequente superioridade da oferta 
em relação à demanda têm evidenciado a importância da inovação para a dinâmica do mercado contemporâneo (CELASCHI, 2007). Assim, "a capacidade de substituir produtos por versões mais modernas" torna-se um verdadeiro fator estratégico dentro das organizações, à medida que diferenciais competitivos passam a ser assegurados por meio do lançamento constante de novos bens de consumo para o mercado (TIDD, PAVITT \& BESSANT, 2008, p.25).

Nesse contexto, o processo relacionado ao desenvolvimento de novos produtos tem despertado o interesse de inúmeros estudos que visam, acima de tudo, compreender quais são os fatores relacionados ao sucesso do projeto (por exemplo: SIMON, 2006; CICMIL, WILLIAMS, THOMAS \& HODGSON, 2006; LEENDERS, ENGELEN \& KRATZER, 2007; BUNDUCHI, 2009). Assim, apesar de existirem algumas contradições, é consenso na maioria dessas análises a importância da geração de espaços que promovam o cruzamento de diferentes conjuntos de conhecimentos a fim de potencializar a criatividade.

Percebe-se que os tradicionais modelos de gestão de projeto não correspondem às necessidades dos projetos que visam desenvolver ambientes criativos. Para Cicmil, Williams, Thomas e Hodgson (2006), os métodos de gerenciamento dos projetos contemporâneos devem ser flexíveis ao invés de inteiramente pré-planejados, ou seja, eles devem ser construídos a partir do próprio processo por meio de trajetórias que sejam favoráveis à criatividade e à inovação. Dessa maneira, ao analisar o processo de desenvolvimento de projetos, Moraes (2010, p.6) constata que

a estrada deve ser sempre projetada e a rota, muitas vezes redefinida durante o percurso. Tudo isso exige dos designers e produtores uma maior capacidade de gestão e maior habilidade na manipulação das informações e mensagens obtidas.

Da mesma forma, o autor destaca que a necessidade de flexibilidade do projeto é o reflexo de um ambiente complexo, marcado pela abundância de informações e pelas constantes mudanças econômicas, sociais e culturais que interferem, diretamente, na forma de pensar e planejar as ações. Nesse sentido, o design, por meio da cultura de projeto, representa uma peçachave na atual dinâmica do mercado, ao passo que seus métodos contemporâneos podem contribuir, significativamente, para a organização de um sistema de informações úteis a fim de potencializar a criatividade durante o processo de desenvolvimento de produtos inovadores (CELASCHI, 2007). Para tanto, a perspectiva do design estratégico sugere a divisão do projeto em duas etapas distintas, sendo uma delas tradicionalmente definida como projeto e, a outra, metaprojeto (CELASCHI, 2007).

Nessa perspectiva, o metaprojeto torna-se uma etapa de grande relevância para o processo de inovação, à medida que estimula reflexões e pesquisas que podem questionar e alterar o briefing. Nessa fase, a pesquisa contextual analisa o cenário no qual o problema está inserido e organiza um sistema de informações pertinentes para o andamento do projeto (DESERTI, 2007). Já a pesquisa blue sky busca construir direções para a inovação através de "uma 'colheita' de elementos úteis para estimular e direcionar a criatividade" (SCALETSKY \& PARODE, 2008, p.2). Scaletsky e Parode (2008) observam que a linguagem visual desempenha um papel bastante relevante para essa modalidade de pesquisa, uma vez que as imagens representam um 
dispositivo capaz de estimular a criatividade e a visualização dos possíveis caminhos para a inovação.

Assim, dentro do metaprojeto, a pesquisa blue sky destaca-se como uma ferramenta que favorece a criação de conceitos e o compartilhamento de ideias e intenções projetuais, fato que vem ao encontro de uma das principais necessidades para o desenvolvimento de novos produtos no contexto do mercado contemporâneo. Tendo isso em vista, o presente artigo objetiva refletir acerca da relevância da pesquisa blue sky durante os processos projetuais que visam a inovação. Para tanto, procurar-se-á compreender de que forma a pesquisa blue sky pode contribuir para o direcionamento criativo do projeto. Da mesma forma, será feita uma reflexão sobre as contribuições dessa pesquisa para o desenvolvimento da criatividade dentro da equipe, assim como em relação à pertinência do uso da linguagem visual durante essa etapa do projeto.

\section{Repensando a gestão dos projetos}

As significativas transformações econômicas, sociais e culturais do final do século XX foram responsáveis pela nova configuração do cenário atual, marcado pela imprevisibilidade e pela grande quantidade de códigos em permanente mutação (MORAES, 2010). Para Moraes (2010, p.6) tal situação configura um ambiente dinâmico, complexo e de difícil compreensão, onde a inovação se faz necessária para "estimular e alimentar constantemente o mercado" que trabalha para diferenciar sua oferta por meio da criação de novos significados.

No entanto, a acirrada competitividade entre as empresas somada à redução do ciclo de vida dos produtos demonstra que não basta inovar, é preciso inovar de forma rápida e alinhada às expectativas de um mercado cada vez mais exigente e instável (LEENDERS, ENGELEN \& KRATZER, 2007). Dessa forma, Leenders, Engelen e Kratzer (2007) constatam que a fase de desenvolvimento dos projetos tende a ser complexa, arriscada e constantemente pressionada por questões relacionadas ao tempo.

Nesse contexto, gerenciar métodos eficazes e capazes de acelerar o processo de desenvolvimento de novos produtos torna-se o novo desafio das empresas contemporâneas que visam, acima de tudo, assegurar vantagens competitivas por meio da inovação. Para tanto, fica cada vez mais clara a importância da criatividade e do uso de métodos capazes de promovê-la. Todavia, Cicmil, Williams, Thomas e Hodgson (2006) observam que os tradicionais modelos de gerenciamento de projetos não estão alinhados a essa nova necessidade projetual. Da mesma forma, Moraes (2010) afirma que a simples abordagem projetual objetiva e linear, então praticada para a concepção dos produtos industriais no passado, não é mais suficiente para garantir o sucesso de uma empresa ou atender à expectativa do usuário (MORAES, 2010, p.13).

Concomitantemente, Williams, Thomas e Hodgson (2006) constatam a necessidade de ampliar as bases intelectuais da disciplina de gerenciamento de projeto para, assim, incluir novas abordagens que vão além da gestão de técnicas padronizadas para a solução de problemas específicos. Assim, pode-se dizer que essa necessidade é o resultado de um novo quadro contextual, onde a gestão da criatividade assume o papel central para o sucesso do desenvolvimento de novos produtos. 
Nesse sentido, Celaschi (2007) observa que a contribuição do design dentro do processo de inovação (convencionalmente vista como funcional) é ampliada para o nível estratégico, surgindo como uma nova alternativa para o gerenciamento dos projetos que visam, por meio de seus processos, alcançar um maior grau de inovação. Paralelamente, "a complexidade hoje presente na atividade de design exige por sua vez, dentro da cultura projetual, a compreensão do conceito da gestão da complexidade", ao passo que o dinamismo dos cenários e o excesso de informações disponíveis requerem o uso de novas ferramentas, instrumentos e metodologias (MORAES, 2010, p.13).

Dessa forma, com o intuito de orientar o processo de concepção e o desenvolvimento de novos produtos em meio a essa complexidade, a inovação que é dirigida pelo design estratégico possibilita dividir o projeto em duas etapas, sendo a primeira delas o metaprojeto e a segunda projeto. Assim, percebe-se que o principal diferencial deste método em relação aos métodos convencionais está na inclusão do metaprojeto, ou seja, na inserção de uma etapa anterior ao projeto que busca realizar uma reflexão acerca do próprio projeto. Para tanto, essa etapa busca realizar uma análise detalhada das variáveis relacionadas ao problema de projeto para, a partir disso, verificar a possibilidade de reposicionar o problema e sugerir possíveis diretrizes para o projeto, apresentadas na forma de um contra-briefing. Nas palavras de Celaschi (2007), o metaprojeto pode ser entendido como o projeto do projeto, uma vez que constitui o plano regulador que norteará todo o processo de inovação.

Na perspectiva de Moraes (2010, p.25), o metaprojeto apresenta-se como uma resposta ao atual "cenário complexo e mutante" onde as metodologias convencionais não estão adaptadas a esta realidade. De acordo com o autor,

o metaprojeto nasce, portanto, da necessidade de existência de uma plataforma de conhecimentos (Pack of tools) que sustenta e orienta a atividade projetual em um cenário fluido e dinâmico que se prefigura em constante mutação. (MORAES, 2010, p.26)

Assim, o metaprojeto compreende, basicamente, a função de organizar as informações e, ao mesmo tempo, estimular o processo criativo (DESERTI, 2007). Em outras palavras, esta seria uma etapa onde diferentes cenários são avaliados através da organização das informações pertinentes para uma reflexão acerca do próprio andamento do projeto. Para tanto, o metaprojeto pode ser dividido em duas fases: a fase de pesquisa contextual e a fase de pesquisa blue sky, conforme destaca o figura 1. 
Figura 1 - Etapa metaprojetual

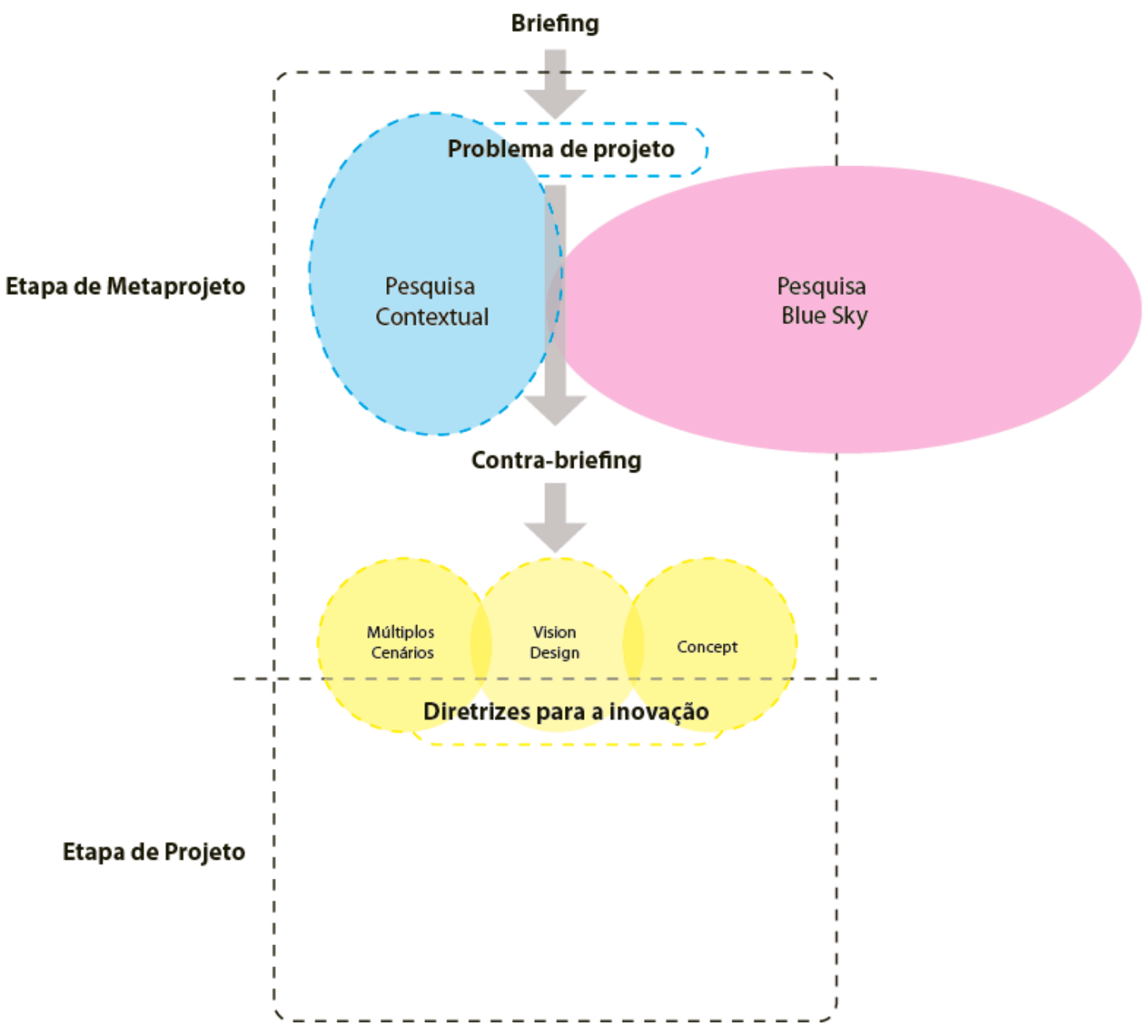

Fonte: desenvolvido pela autora.

Com base nisso, o presente estudo volta-se à análise e compreensão da pesquisa blue sky, a qual desenvolve métodos de estímulo à criatividade e inovação.

\section{Pesquisa Blue sky: criatividade para o processo de inovação}

Inserida na fase metaprojetual do design estratégico, a blue sky pode ser entendida como uma pesquisa não estruturada que objetiva organizar uma série de estímulos para o desenvolvimento de novos produtos (CAUTELA, 2007, p.2). A blue sky proporciona a construção de um "sistema de informações" capaz de auxiliar o processo criativo inerente aos projetos que 
envolvem inovação (MORAES, 2010, p.42).

Leenders, Engelen e Kratzer (2007) constatam que a criatividade em equipes de desenvolvimento de novos produtos é estimulada através da eficácia do intercâmbio de informação. Para os autores, é com base no conhecimento dos outros que os membros da equipe podem fazer a fertilização cruzada das ideias a fim de gerar novos conhecimentos e compreensões que viabilizam o andamento do projeto. Assim, percebe-se que a pesquisa blue sky pode ser uma alternativa favorável à esse intercâmbio de informações, à medida que seus métodos contribuem para a organização de todos os dados levantados, assim como para o compartilhamento dos conhecimentos pertinentes aos processo.

De acordo com Scaletsky e Parode (2008),

a construção de pesquisas blue sky poderia ser associada a um processo de busca e externalização de um conhecimento tácito trazido pelos designers que trabalham sobre um projeto, decorrente de suas experiências e modelos mentais (SCALETSKY \& PARODE, 2008, p.2).

Ao analisar os mecanismos de transformação do conhecimento tácito em coletivo nas equipes de desenvolvimento de novos produtos, Hirunyawipada, Beyerlein e Blankson (2010) constatam que a congruência dos objetivos é capaz de aumentar a capacidade da equipe em alcançar um estado mental compartilhado e de entendimento conjunto, favorecendo, assim, a transformação do conhecimento tácito em coletivo. Diante disso, observa-se que a pesquisa blue sky também pode ser um meio pertinente para alcançar essa congruência, pois facilita a expressão individual e a exposição de ideias que, após uma reflexão conjunta, adquirem significados comuns à todos os membros da equipe.

Assim, é por meio da construção de significados claros e conhecimentos compartilhados que a pesquisa blue sky pode auxiliar na construção do que Deserti (2007) chama de trajetórias de inovação. Da mesma forma, observa-se que, diferente da pesquisa contextual, a blue sky "não mantém um vínculo de dependência direta em relação ao problema", ampliando o olhar do projetista para outros contextos de referências diferentes daqueles diretamente ligados às questões do projeto, fato que favorece, mais uma vez, o desenvolvimento da inovação (SCALETSKY \& PARODE, 2008, p.2).

Para Scaletsky e Parode (2008, p.2) a blue sky incentiva os processos chamados "crossfertilization", ou seja, os processos que buscam potencializar a criatividade através de estímulos à intuição dos projetistas na busca de suas referências. Entretanto, Cautela (2007) observa que esse contexto criativo deve gerar reflexões que contribuam para direcionar a inovação e o desenvolvimento do projeto. Com isso, pode-se dizer que, mesmo sendo uma pesquisa aberta e não estruturada, a blue sky deve ter como objetivo a construção de estímulos que sejam realmente úteis para o andamento do projeto. Dessa forma, apesar de os problemas de projeto não serem abordados de forma direta, eles não devem ser totalmente ignorados, muito pelo contrário, eles devem aparecer de forma implícita durante todo o processo de desenvolvimento da pesquisa blue sky. 
Assim, percebe-se na blue sky uma importante pesquisa metaprojetual que procura auxiliar a capacidade da equipe de projeto de "ver" e "fazer ver" os fenômenos que estão muito além da superfície visível pelos processos de projeto convencionais. Essa modalidade de pesquisa contribui para orientar a visão sobre os objetivos apresentados, bem como para acelerar as tomadas de decisões através da exposição clara dos pensamentos criativos por meio do uso de ferramentas como moodboards e storyboards (ZURLO, 2010). Dessa forma, Deserti (2007) constata que é bastante comum o uso de imagens nessa pesquisa, ou seja, de uma linguagem visual capaz de contribuir para a criação e visualização dos novos significados desenvolvidos nesse processo.

\section{4 uso da linguagem visual na pesquisa Blue Sky}

A linguagem visual pode ser compreendida como uma estrutura de formas visuais organizadas pelo ser humano na forma de linguagem, isto é, se refere à maneira como as formas visuais são representadas e adquirem sentido (SANTAELLA, 2005). No design, o uso da linguagem visual adquire papel central, auxiliando tanto na construção dos processos criativos como na comunicação das ideias e de seus significados. No caso da pesquisa blue sky, o uso da linguagem visual é bastante frequente, sendo que "uma imagem nessa perspectiva é um dispositivo, um signo produtor de sentido" capaz de construir conceitos e visões favoráveis ao projeto (SCALETSKY \& PARODE, 2008, p.1).

Santaella (2008) observa que as imagens estabelecem significados por meio da união do que a autora define como domínios. Assim, o primeiro domínio compreende as imagens na forma de representações visuais que podem ser percebidas através de desenhos, pinturas, fotografias, etc. Imagens, nesse sentido, são objetos materiais, são signos que representam algo no ambiente visual. Já o segundo domínio corresponde à forma imaterial da imagem, isto é, as imagens da mente humana. Segundo a mesma autora, nesse domínio, as imagens aparecem na forma de visões, fantasias, esquemas, ou seja, como representações mentais.

Com isso, observa-se que a blue sky é uma pesquisa que possibilita a externalização das representações mentais através da busca de imagens (ou seja, representações visuais) que sejam representativas desses modelos mentais. Tendo isso em vista e retomando os estudos de Hirunyawipada, Beyerlein e Blankson (2010) constata-se que o uso da linguagem visual pode contribuir, significativamente, para a transformação do conhecimento tácito em coletivo, à medida que essa linguagem facilita a comunicação e compreensão das ideias que estão internalizadas na mente do projetista e que, futuramente, poderão guiar o processo de inovação.

Ao mesmo tempo, a materialização de representações metais contribui para a organização do pensamento que se encontra mergulhado na complexidade do cenário contemporâneo. Dessa forma, torna-se possível "ver" as próprias ideias e, ao mesmo tempo, conectar ideias que talvez, com o uso de outros métodos, ficariam presas separadamente na mente de cada projetista. Com isso, pode-se dizer que a blue sky contribui favoravelmente para o que Leenders, Engelen e Kratzer (2007) chamam de fertilização cruzada das ideias.

Paralelamente, a linguagem visual também promove a construção de metáforas criativas que viabilizam a criação de novos significados que são fundamentais para o processo de inovação 
(SCALETSKY \& PARODE, 2008). Nas palavras de Scaletsky e Parode (2008),

ao agrupar as imagens segundo uma lógica específica para cada contexto, a pesquisa blue sky cria novos significados que vão além das imagens e palavras nela contidas (SCALETSKY \& PARODE, 2008, p.2).

Com isso, constata-se que o uso de imagens na pesquisa blue sky facilita o "fazer ver" tanto à nível de equipe de projeto (favorecendo a já mencionada congruência dos objetivos) como à nível de construção e visualização dos conceitos que irão nortear todo o processo de inovação.

\section{Considerações Finais}

A revisão teórica permite afirmar que a relevância da inovação para a dinâmica do mercado contemporâneo exige a implementação de métodos diferenciados para o desenvolvimento de novos produtos, com o intuito de criar contextos criativos e favoráveis para os processos de inovação. Com isso, a gestão do projeto deve abranger a gestão da criatividade e da própria complexidade em que se insere o projeto, à medida que o cenário atual é marcado pelo grande volume de informações e pelas constantes transformações sociais, culturais e econômicas que afetam diretamente a maneira de pensar o próprio projeto.

Nesse sentido, o design, por meio da cultura de projeto, destaca-se como uma alternativa pertinente para o direcionamento dos projetos, uma vez que seus métodos e ferramentas possibilitam a criação de um sistema de informações úteis, ou seja, de um sistema que organiza essa complexidade e que, ao mesmo tempo, colabora para direcionar a criatividade durante o processo de desenvolvimento de produtos inovadores. Para tanto, a perspectiva do design estratégico sugere a divisão do projeto em duas etapas: o metaprojeto e o projeto.

Nessa perspectiva, percebe-se que o metaprojeto é compreendido como uma fase de reflexão acerca do próprio projeto e, por meio da pesquisa blue sky, desempenha um papel importante para o processo de inovação, considerando que confere ao projeto um campo bastante fértil para a exploração da criatividade. Para tanto, a blue sky busca estímulos (referências) que vão além daqueles diretamente ligados ao problema do projeto. Ao mesmo tempo, percebe-se que, em muitos casos, esses estímulos são imagens selecionadas como referência para representar ideias e significados. Nesse sentido, destaca-se aqui que uma das principais contribuições da pesquisa blue sky para o processo de desenvolvimento de um sistema-produto inovador está na sua capacidade de favorecer a comunicação entre a equipe, colaborando para a externalização do conhecimento tácito e consequente cruzamento de ideias que, frequentemente, tendem à favorecer a criatividade. Dessa forma, considera-se que a blue sky é uma fase relevante aos projetos que visam a inovação, ao passo que é uma pesquisa voltada para a construção de um ambiente criativo que busca definir trajetórias inovadoras.

\section{Referências}


BUNDUCHI, R. Implementing Best Practices to Support Creativity In NPD Cross-Function Teams. International Journal of Project Management, 2009.

CAUTELA, Cabirio. Strumenti di design management. Milão: Franco Angeli, 2007, p. 95-97.

CELASCHI, F. Dentro al progetto: a ppunti di merceologia contemporánea, in: Celaschi, F.; Deserti, A. Design e innovazione: strumenti e pratiche per la recerca aplícate. Carocci, Roma, 2007.

CICMIL, S.; WILLIAMS, T.; THOMAS, J,; HODGSON, D. Rethinking project management: researching the actuality of projects. International Journal of Project Management, 2006.

DE MORAES, Dijon. Metaprojeto: o design do design. São Paulo: Edgard Blucher, 2010.

DESERTI, A. Intorno al progetto: concretizzare innovazione in: Celaschi, F.; Deserti, A. Design e innovazione: strumenti e pratiche per La ricerca applicata. Carocci, Roma, 2007.

HIRUNYAWIPADA, $t$,; BEYERLEIN, M.; BLANKSON, C.. Cross-function integration as a knowledge transformation mechanism: Implications for new product development. Industrial Marketing Management, 2010.

LEENDERS, R.; VAN ENGELEN, J.; KRATZER, J. Systematic Design Methods and the Creative Performance of New Product Teams: Do They Contradict or Complement Each Other? Journal of Product Innovation Management, 2007.

SANTAELLA, Lucia. Imagens: cognição, semiótica, mídia. 1.ed. São Paulo: Iluminuras: FAPESP, 2008.

SCALETSKY, C.; PARODE, F.P.. Imagem e pesquisa Blue sky no design. Convención Científica de ingeniería y arquitectura. La Bahana, 2008.

SIMON, L. Managing creative projects: An empirical synthesis of activities. International Journal of Project Management, 2006.

TIDD, J.; PAVITT, K.; BESSANT, J. Gestão da Inovação. Porto Alegre: Bookman, 2008.

ZURLO, Francesco. Design Strategico, in AA. VV., Gli Spazi e le arti, Volume IV, Opera XXI Secolo, Editore Enciclopédia Treccani, Roma, 2010. 